Trauma Berufskrankh 2009 · 11

[Suppl 1]:122-127

DOI 10.1007/s10039-008-1420-y

Online publiziert: 13. September 2008

(c) Springer Medizin Verlag 2008

A. Partenheimer $\cdot$ J. Geerling $\cdot$ H. Lill

Klinik für Unfall- und Wiederherstellungschirurgie, Abteilung des BG-Krankenhauses Hamburg im Friederikenstift, Diakoniekrankenhaus Friederikenstift Hannover gGmbH, Hannover

\title{
Fehlbeurteilung und -versorgung von Fußverletzungen
}

tomographie (CT) und Magnetresonanztomographie (MRT) erforderlich.

Auch bei der intraoperativen Diagnostik sollte die Indikation zur intraoperativen Iso-C-3D-Bildgebung (Iso-C-3D: mobiler chirurgischer Bildverstärker) weit gestellt werden. Durch sie können in bis zu 39\% der Fälle eine intraoperative Fehllage des Implantats oder eine Fehlreposition erkannt und eine Korrektur vorgenommen werden [4].

\section{Talus}

Die Talusfraktur ist mit einen Anteil von etwa 3-6\% aller Fußverletzungen sehr selten [1]. Es werden die zentralen von den peripheren Frakturen unterschieden. Erstere entstehen typischerweise durch axiale Stauchung bei dorsal extendiertem Fuß und bei beträchtlicher Krafteinwirkung, wie es häufig im Rahmen von Verkehrsunfällen der Fall ist. Die besondere Form der Gefäßversorgung ist für die Prognose der zentralen Talusfraktur entscheidend. Von der Form der Fraktur sind das Risiko der posttraumatischen Durchblutungsstörung und damit die Pseudarthrosenrate abhängig [4, 5, 14].

Im Wesentlichen wird die Prognose der zentralen Talusfraktur vom Frakturverlauf und vom Ausmaß der Dislokation bestimmt. Das Nekroserisiko wird für zentrale Trümmerfrakturen mit 1:1,8, für Hawkins-III-Frakturen mit 1:2, für Hawkins-I-Frakturen mit 1:10 angegeben [24]. Nach einer Untersuchung von Kundel et al. [11] wurde global gesehen nur bei
15\% der Patienten eine völlige Wiederherstellung der Funktion erreicht. 34\% der Patienten zeigten eine Arthrose im oberen und/oder unteren Sprunggelenk. 55\% der Patienten dieser Serie hatten Beschwerden, ohne eine Arthrose oder Nekrose erkennen zu lassen [9].

Neben posttraumatischen Nekrosen des Talus führen auch immer wieder periphere Frakturen zu Fehlinterpretationen. Dabei handelt es sich um Brüche der Gelenkflächen, des Processus sowie von Taluskopf und peripherem, also kopfnahem Talushals.

\section{Kalkaneus}

Auch bei den Kalkaneusfrakturen kann man die zentralen von den peripheren Brüchen unterscheiden.

Zentrale Frakturen. Die wohl wichtigste und am häufigsten angewandte Klassifikation ist diejenige von Essex-Lopresti [6], die die zentralen Kalkaneusfrakturen entsprechend ihrer Form einteilte in:

- „tongue type“ und

- „joint depression type“.

Bei diesen zentralen Brüchen kommt es häufig zu postoperativen Fehlstellungen mit resultierender Fehlstatik des Fußes oder zu posttraumatischen Arthrosen im Bereich des unteren Sprunggelenks.

Periphere Frakturen. Sie werden in den Standardaufnahmen des Fußes bzw. des 
Sprunggelenks häufig fehlinterpretiert oder übersehen.

Generell ist bei der Behandlung von Kalkaneusfrakturen in erster Linie ein adäquates Weichteilregime für ein gutes Ergebnis essenziell. Zudem ist eine anatomische Rekonstruktion des gesamten Kalkaneus mit einer anschließenden funktionellen Weiterbehandlung zu fordern. Bei 2-Teile-Frakturen nach der Sanders-Klassifikation kann in mehr als $80 \%$ der Fälle eine anatomische Rekonstruktion erzielt werden [20]. In Anbetracht der bestehenden Knorpelschädigung durch das initiale Trauma ist ein gutes oder sehr gutes Ergebnis bei etwa 70\% der Patienten möglich. Bei 3-Teile-Frakturen kann die anatomische Reposition zwar nur in etwa $60 \%$ der Patienten gelingen, doch sind auch hier $70 \%$ insgesamt gute Ergebnisse eine realistische Zielvorstellung [21].

Eine verzögerte Knochenheilung kann durch den initialen Weichteilschaden bedingt sein, aber auch durch schlechte Knochenqualität und fehlende Belastung.

Unter Berücksichtigung der technischen Ansprüche bei der operativen Rekonstruktion von Kalkaneusfrakturen ist zu folgern, dass diese von Spezialisten durchgeführt werden sollte.

\section{Vordere Fußwurzel}

Sie wird vom Os naviculare, Os cuboideum und den 3 Ossae cuneiforme I-III gebildet. Die Verletzungen der einzelnen Knochen entstehen aufgrund ihrer anatomischen Beziehung zueinander und $\mathrm{zu}$ den mitbeteiligten Strukturen [7, 22]. Beim Os naviculare handelt es sich hierbei um Avulsions- und Körperfrakturen, beim Os cuboideum um die so genannte Nussknackerfraktur, die bei forcierter Vorfußabduktion entstehen kann, und bei den Verletzungen der Ossae cuneiforme um Kompressionsfrakturen, welche häufig auch mit Luxationsformen im Chopart-Gelenk assoziiert sind $[18,19]$.

Aufgrund der engen Beziehung der Knochen zueinander werden Verletzungen im Bereich der Fußwurzel in den Standardaufnahmen des Mittelfußes in 2 Ebenen häufig übersehen. Diagnostiziert werden sie in solchen Fällen erst durch eine massive Klinik mit ausgeprägter Weichteilschwellung oder aufgrund per- sistierender Beschwerden, die dann zu einer Ausweitung der Diagnostik führen $[16,24]$.

\section{Chopart- und Lisfranc-Luxationsfrakturen}

Neben den isolierten Brüchen gehören auch Luxationen und/oder Luxationsfrakturen des Chopart- bzw. Lisfranc-Gelenks zu den typischen Verletzungen. Insbesondere Luxationsfrakturen stellen häufig folgenschwere Läsionen dar, die sich im weiteren Verlauf auf die gesamte Fußfunktion auswirken können $[2,3,8,9]$.

Eine hohe Anzahl an kombinierten Verletzungen der Fußwurzel wird primär übersehen. Aus diesem Grund ist die Primärdiagnostik bereits beim geringsten Verdacht auszudehnen. Neben den Standardröntgenaufnahmen des Fußes in 3 Ebenen muss bei entsprechendem Verdacht die Indikation für die Computertomographie inklusive multiplanarer Rekonstruktion großzügig gestellt werden. Im Gegensatz zum CT sollte die MRT speziellen Fragestellungen vorbehalten bleiben [15].

Die Grundprinzipien der adäquaten Behandlung sind die anatomiegerechte Rekonstruktion der Gelenkstrukturen und der geometrischen Beziehungen der medialen zur lateralen Fußsäule sowie die Erfassung und Therapie der ligamentären Instabilitäten. Werden diese Ziele nicht erreicht oder treten Komplikationen auf, sind oft Korrekturarthrodesen unvermeidlich $[13,23]$.

\section{Metatarsalia}

Frakturen derselben sind mit 30\% aller Fußverletzungen die häufigsten Brüche des Fußes. Von Bedeutung sind v. a. die Metatarsale-I- und -V-Frakturen, da diese nach dem 3-Pfeiler-Modell die Basis für die Fußstatik darstellen [17].

\section{Metatarsale I}

Es ist für das physiologische Abrollverhalten von besonderer Bedeutung. Deshalb sollte die konservative Behandlung hier nur den einfachen, nicht dislozierten Frakturen vorbehalten bleiben. Bereits leicht verschobene Frakturen stel-
Trauma Berufskrankh 2009 · 11

[Suppl 1]:122-127

DOI 10.1007/s10039-008-1420-y

C) Springer Medizin Verlag 2008

A. Partenheimer $\cdot$ J. Geerling $\cdot$ H. Lill

Fehlbeurteilung
und -versorgung
von Fußverletzungen

Zusammenfassung

Die komplexe Anatomie mit der im Verhältnis zur Knochenanzahl häufigen Gelenkbeteiligung ist für die Behandlung von Fußverletzungen von grundlegender Bedeutung. So ergeben sich zum einen schwierige Osteosyntheseverhältnisse, zum anderen aufgrund der geringen Weichteildeckung hohe Anforderungen an den Operateur. Durch Fehlinterpretation oder -behandlung kann es zu posttraumatischen Arthrosen und pseudarthrotischen Zuständen kommen. Grundlegend in der Behandlung von Fußverletzungen ist die ausgedehnte Diagnostik, die über die initial durchgeführten Standardröntgenaufnahmen hinaus erweitert werden muss. Da die Inzidenz von isolierten Fußverletzungen als niedrig einzustufen ist, bedarf es einer ausgewiesenen Expertise des Operateurs und ggf. einer Weiterleitung des Patienten in ein ausgewiesenes Zentrum.

\section{Schlüsselwörter}

Fußverletzung · Beurteilung - Osteosynthese . Posttraumatische Arthrose - Pseudarthrose

\section{Incorrect assessment and treatment of foot injures}

\section{Abstract}

The complex anatomy and ratio of joint participation in relation to the number of bones is of fundamental importance for the treatment of foot injuries. Thus on the one hand difficult osteosynthesis conditions result, while on the other low soft tissue coverage makes high demands on the operating surgeon. Misinterpretation or mistreatment can lead to posttraumatic arthrosis and pseudarthrosis. In the treatment of foot injuries, extensive diagnostics which reach beyond initial standard radiographs are fundamental if later complications are to be avoided. Since the Incidence of isolated foot injuries is low, the operating surgeon requires proven expertise or the patient should be referred to a specialized center if necessary.

\section{Keywords}

Foot injury · Assessment - Osteosynthesis . Posttraumatic arthrosis . Pseudoarthrosis 


\section{Der vermeintlich einfache Fall}
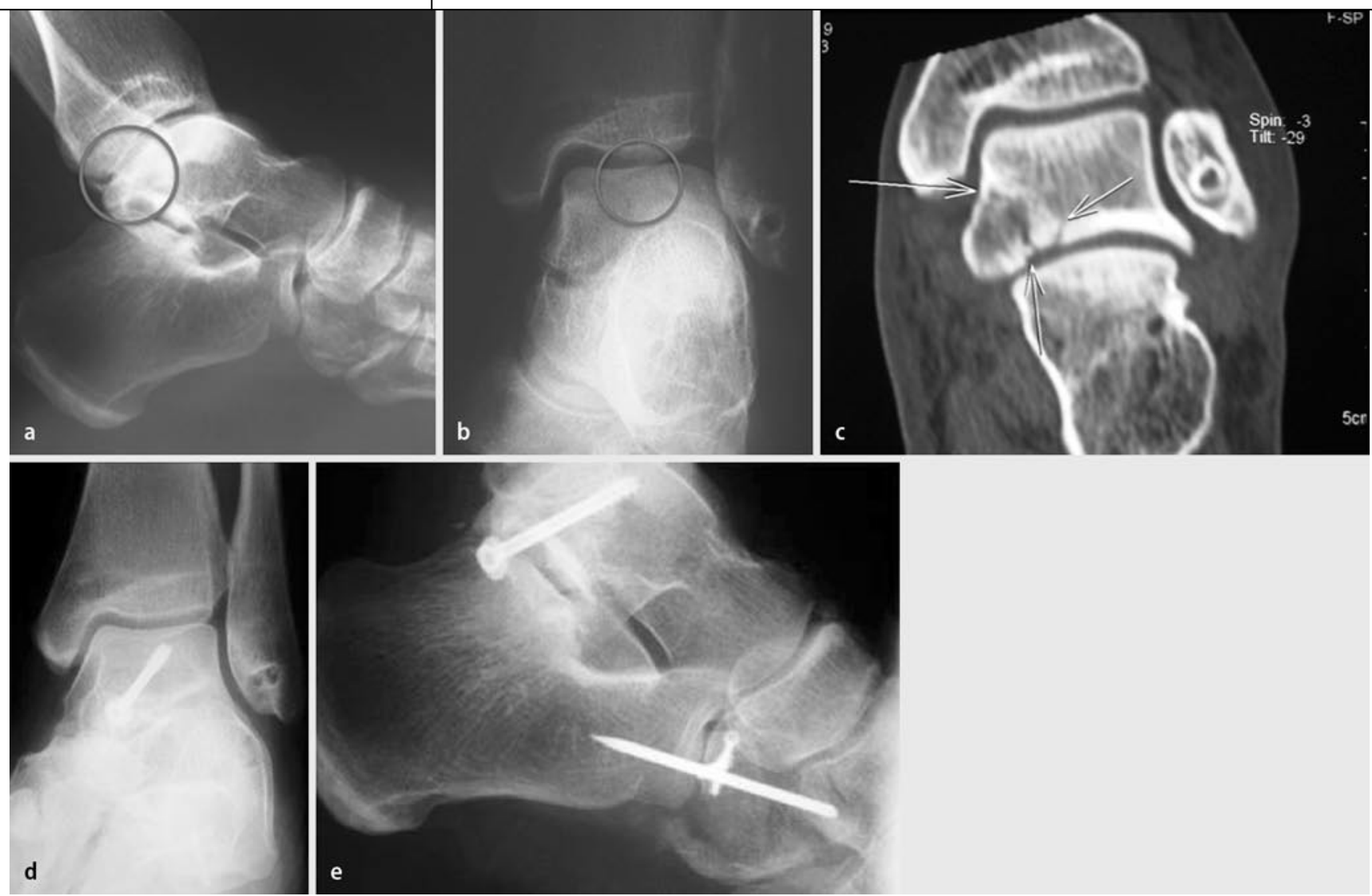

Abb. $1 \Delta$ Fall 1, Sekundärverlegung bei Verdacht auf Chopart-Luxation, a,b Nativröntgenaufnahmen, c CT-Scan, d,e postoperative Versorgungsbilder mit temporärer Arthrodese des kalkaneokuboidealen Gelenks, Erläuterungen s. Text

len eine Operationsindikation dar, können aber minimalinvasiv mittels intramedullärer Kirschner-Drahtung behandelt werden. Höhergradige Verletzungen bedürfen hingegen der offenen Reposition und internen Fixation mittels Plattensystemen. Da am Metatarsale I nur eine geringe Weichteildeckung besteht, gilt es auch hier, ein differenziertes Weichteilregime einzuhalten. Bei regelrechter Reposition und adäquatem Weichteilmanagement heilen diese Verletzungen in aller Regel folgenlos aus.

\section{Metatarsale V}

Frakturen desselben werden seit 1983 nach Lawrence u. Botte [12] eingeteilt in:

- Avulsionsfrakturen,

- Jones-Frakturen und

- Schaftfrakturen.

Avulsionsfrakturen können bei nur geringer Dislokation konservativ behandelt werden. Bei den Jones-Frakturen kommt es trotz ausreichender Ruhigstellung und korrekter Reposition häufig zu Pseud- arthrosen, sodass hier primär die operative Therapie durchgeführt werden sollte. Schaftfrakturen stellen wie die Metatarsale-I-Frakturen bei Dislokation und Mehrfragmentfrakturen eine Operationsindikation dar.

\section{Kasuistik}

\section{Fall 1}

Bei der 31-jährigen Patientin mit Sekundärverlegung bei Verdacht auf Chopart-Luxation war es nach axialem Stauchungstrauma zu einer massiven Schwellung des Mittelfußes und des Sprunggelenks gekommen.

Im Nativröntgenbild ergab sich der Verdacht auf eine Talusfraktur ( $\mathbf{D}$ Abb. 1a, b), die im CT-Scan bestätigt werden konnte (- Abb. 1c). Postoperative wurde eine temporäre Arthrodese des kalkaneokuboidealen Gelenks durchgeführt (• Abb. 1d, e).
Fall 2

Die 20-jährige Patienten hatte nach einen Supinationstrauma eine starke Schwellung des Mittelfußes entwickelt. Die nach initialer konventioneller Röntgenuntersuchung (- Abb. 2a, b) durchgeführte CTUntersuchung mit Reformation zeigte eine Abrissfraktur des Proc. anterior calcanei (- Abb. 2c, d), die operativ versorgt wurde (- Abb. 2e, f).

\section{Fall 3}

Der 45-Jährige stellte sich nach einem Sprung aus $2 \mathrm{~m}$ Höhe initial wegen Schwellung und Schmerzen des Mittelfußes vor.

Im initialen Röntgenbild fand sich kein eindeutiges pathologisches Korrelat (• Abb. 3a). Computertomographisch Horizontalschnitt und 3 D-Reformation konnte eine Chopart-Luxation diagnostiziert werden, die operativ behandelt wurde (- Abb. 3b, c). 

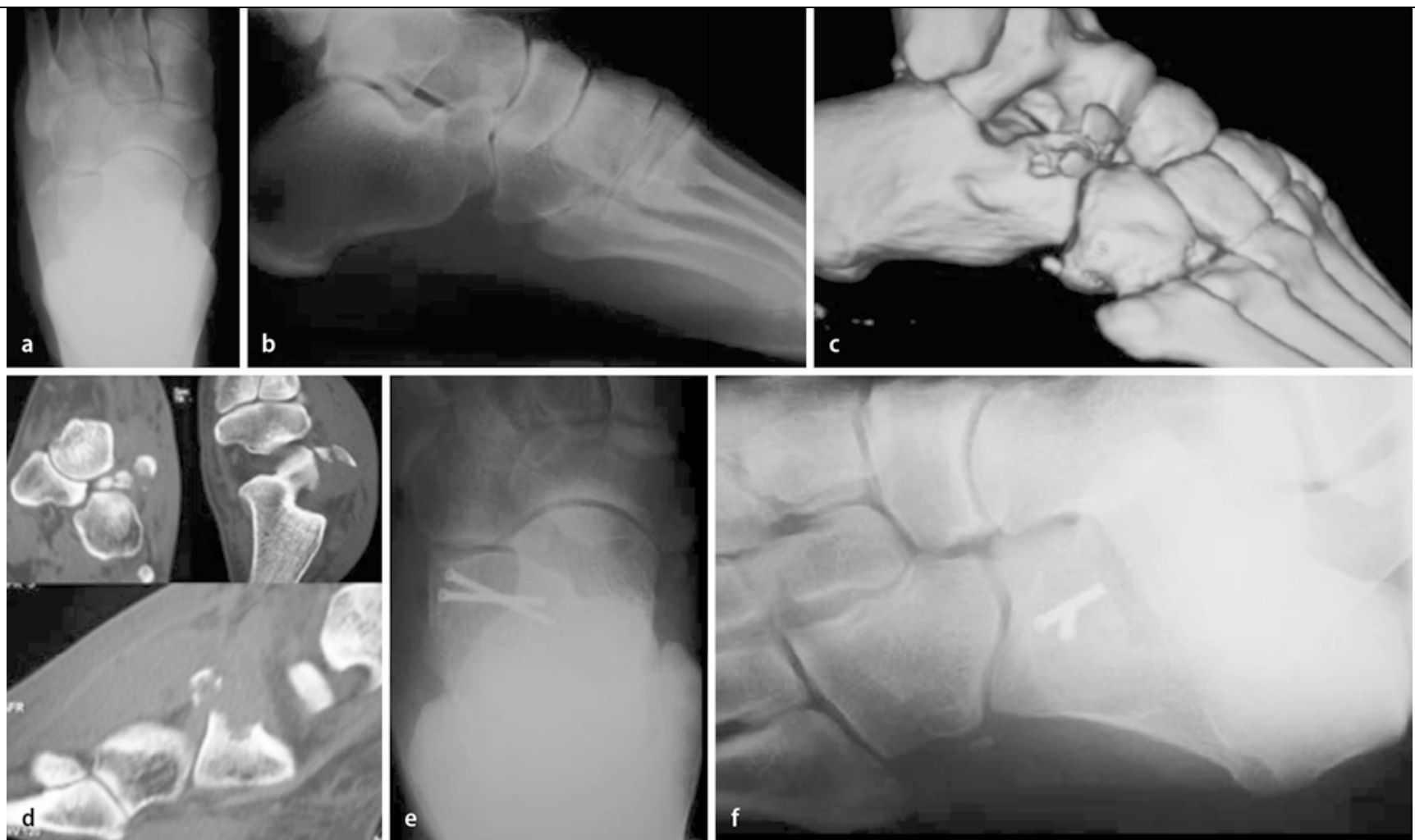

Abb. $2 \Delta$ Fall 2, Supinationstrauma des Fußes, a,b initiale konventionelle Röntgenbilder, c,d CT mit Reformation: Abrissfraktur des Proc. anterior calcanei, e,f postoperative Röntgenaufnahmen in 2 Ebenen, Erläuterungen s. Text

Abb. $3 \triangleright$ Fall 3, a initiale Röntgenbilder, $\mathbf{b}$ CT-Horizontalschnitt und -3D-Reformation, c postoperative Versorgung, Erläuterungen s. Text
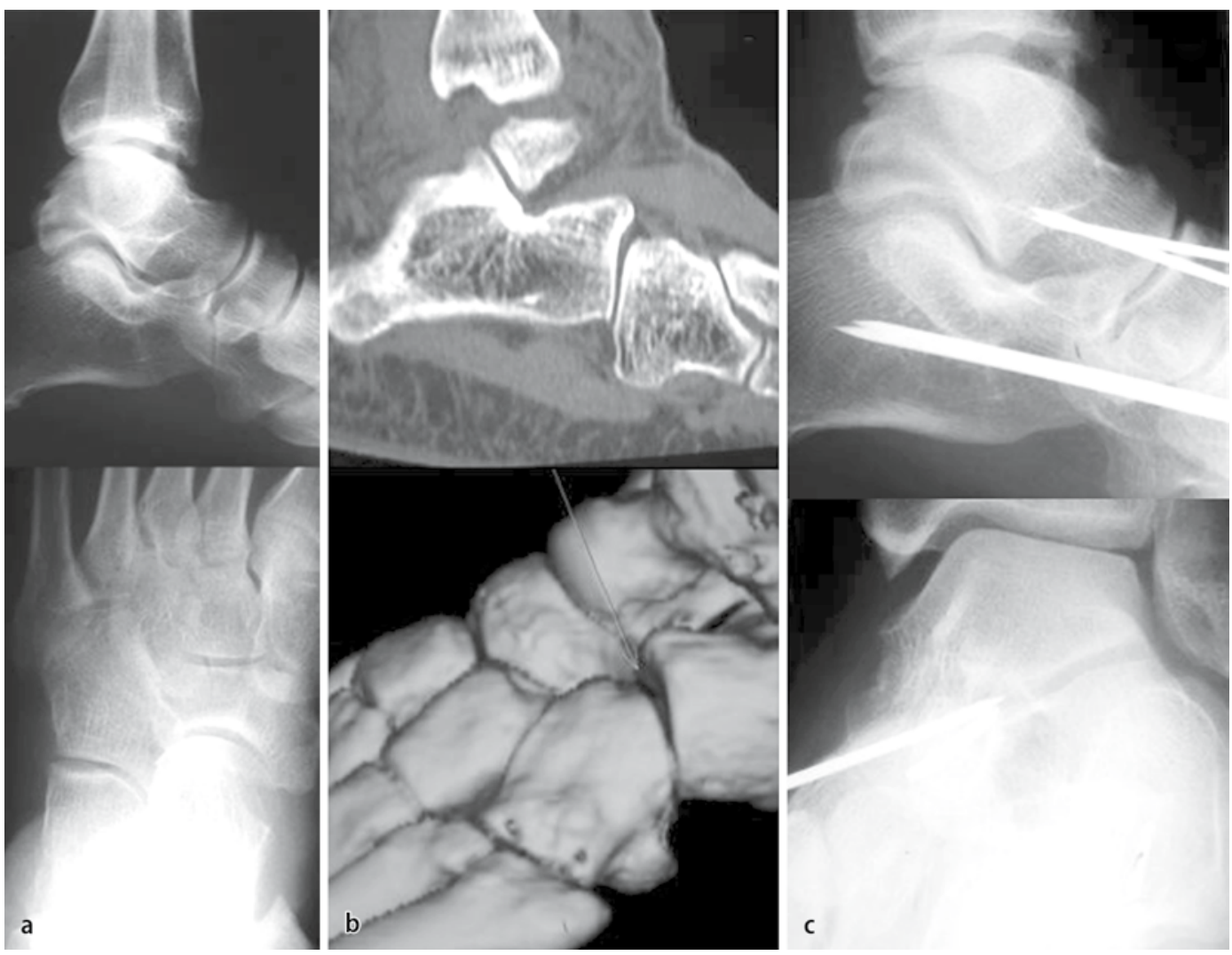


\section{Der vermeintlich einfache Fall}
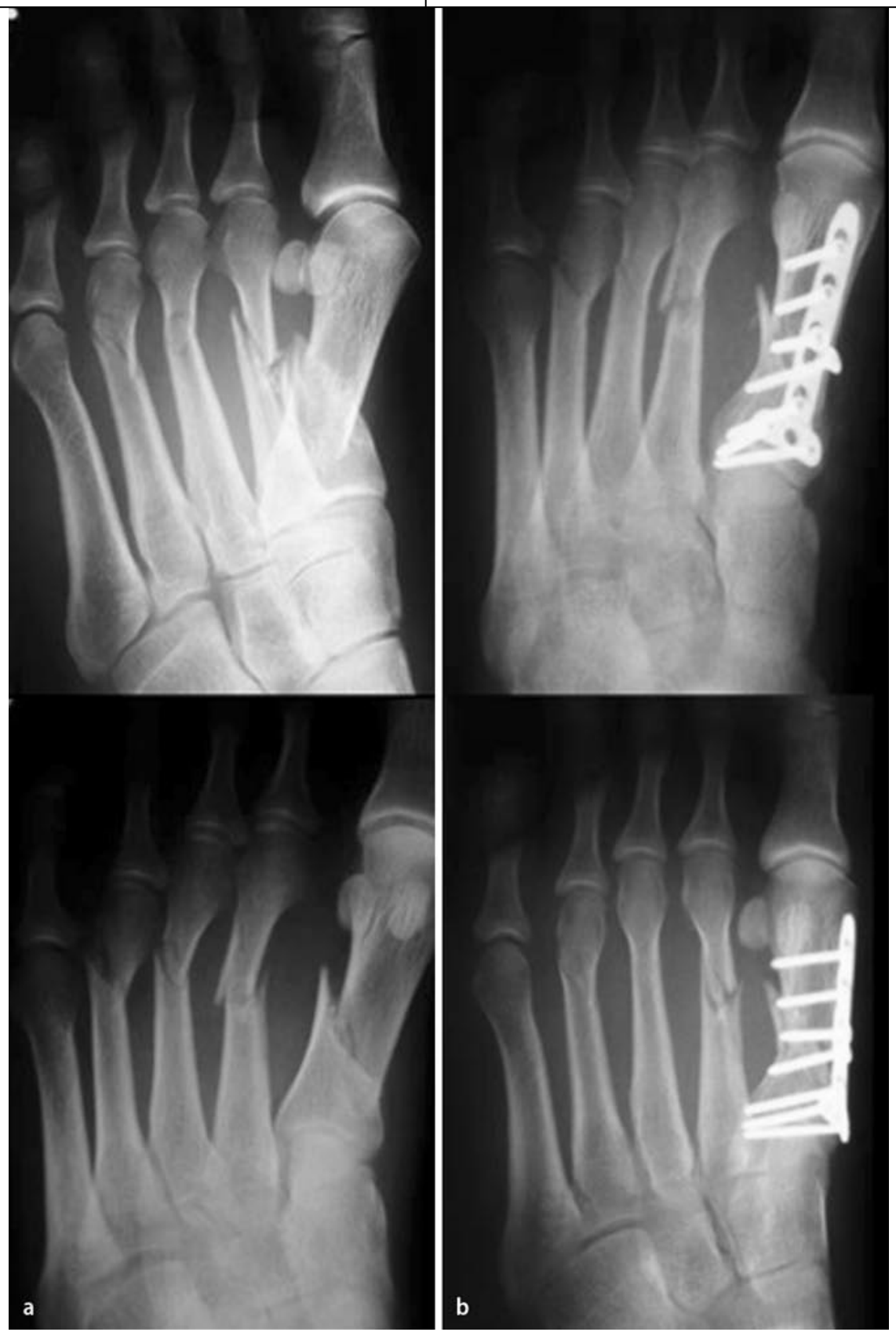

Abb. 4 A Fall 4, a initiale Röntgenbilder, b postoperativ nach ORIF („open reduction, internal fixation“) des Metatarsale I, Erläuterungen s. Text

\section{Fall 4}

Die 27-jährige Frau stellte sich nach axialem Stauchungstrauma vor. In den initialen Röntgenbildern konnte eine Metatarsalia-I-IV-Fraktur diagnostiziert werden. Durch ORIF („open reduction, internal fixation") des Metatarsale I wurde eine regelrechte Stellung der Metatarsalia II-IV ohne Rotations-/Längenfehlstellung erzielt (• Abb. 4).

\section{Fazit}

Verletzungen des Fußes sind selten. Häufig sind sie in den initial durchgeführten Standardröntgenaufnahmen schwer erkennbar und werden oft übersehen. Um dies zu verhindern, gilt es, eine ausreichende Diagnostik mit Spezialaufnahmen des Fußes in 3 Ebenen durchzuführen und die Indikation zum CT großzügig zu stellen. Unabdingbar bei Verletzungen des Fußes sind auch die adäquate Beurteilung und Behandlung der begleitenden Weichteilverletzungen. Bei Frakturnachweis im Bereich der Fußwurzel sind ein präoperatives CT und/oder ein intraoperatives Iso-C-3D zu empfehlen. Die dislozierten Frakturen der Fußwurzel stellen eine Operationsindikation dar. Bei der Versorgung der Brüche sind eine Rekonstruktion der Anatomie der verletzten Fußpartie und die anatomische Reposition der Gelenkflächen zu fordern. Bei Pseudarthrosen und Fehlstellungen sollte primär die gelenkerhaltende Korrektur angestrebt werden. Ist dies nicht mehr möglich, stellen sich die Single-Arthrodesen oder, nach ausgiebiger Analyse und in seltenen Fällen, auch die Totalendoprothesenimplantation als Alternative dar. Die Frakturen der Metatarsalia haben insgesamt eine günstige Prognose. Eine Ausnahme stellen Chopart- und Lisfranc-Luxations-Frakturen dar, deren Behandlung sich als besonders schwierig erweisen kann. Bei der operativen Versorgung müssen relevante Verkürzungen und Achsenabweichungen vermieden werden.

\section{Korrespondenzadresse}

\section{Dr. A. Partenheimer}

Klinik für Unfall-

und Wiederherstellungschirurgie,

Abteilung des BG-Krankenhauses Hamburg

im Friederikenstift, Diakoniekrankenhaus

Friederikenstift Hannover gGmbH,

Humboldstraße 5, 30169 Hannover

axel.partenheimer@friederikenstift.de

Interessenkonflikt. Der korrespondierende Autor gibt an, dass kein Interessenkonflikt besteht.

\section{Literatur}

1. Adelaar RS (1989) The treatment of complex fractures of the talus. Orthop Clin North Am 20: 691707

2. Arntz CT, Veith RG, Hansen ST (1988) Fractures and fractur-dislocations of the tarsometatarsal joint. $J$ Bone Joint Surg Am 70: 173-181

3. Brunet JA, Wiley JJ (1987) The late results of tarsometatarsal joint injuries. J Bone Joint Surg Br 69: 437-440

4. Canale ST, Kelly FB jr (1978) Fractures of the neck of the talus: longterm evaluation of seventy-one cases. J Bone Joint Surg Am 60A: 143-156

5. Darder-Prats AD, Silvestre-Munnoz A, Suegura-Llopis F et al. (1993) Surgery for fracture of the calcaneus - five year follow-up of twenty cases. Acta Orthop Scand 64: 161-164

6. Essex-Lopresti P (1952) The mechanism, reduction technique and results and fractures of the os calcis. Br J Surg 39: 395-399 
7. Goossens M, De Stoop N (1993) Lisfranc's fracture-dislocations: etiology, radiology and results of treatment. Clin Orthop 176: 154-162

8. Hansen jr ST (2000) Functional reconstruction of the foot and ankle. Lippincott Williams \& Wilkins, Philadelphia

9. Hardcastle PH, Reschauer R, Kutscha-Lissberg E et al. (1982) Injuries to the tarsometatarsal joint. J Bone Joint Surg Br 64: 349-356

10. Hawkins LG (1970) Fractures of the neck of the talus. J Bone Joint Surg Am 52: 991-1002

11. Kundel K, Braun W, Scherer A (1995) Late results of central talus injuries. Unfallchirurg 98: 124-129

12. Lawrence SJ, Botte MJ (1993) Jones' fractures and related fractures of the proximal fifth metatarsal. Foot Ankle 14: 358-365

13. Ly TV, Coetzee JC (2006) Treatment of primary ligamentous Lisfranc joint injuries: primary arthrodesis compared with open reduction and internal fixation: a prospective, randomized study. J Bone Joint Surg Am 88-A: 514-520

14. Marti R (1978) Talus- und Calcaneusfrakturen. In: Weber BG, Brunner C, Freuler F (Hrsg) Frakturbehandlung bei Kindern und Jugendlichen. Springer, Berlin Heidelberg New York, S 376-S387

15. Peicha G, Preidler KW, Lajtai G et al. (2001) Diagnostische Wertigkeit von Nativröntgen, Computer- und Magnetresonanztomographie beim akuten Hyperflexionstrauma des Fußes. Eine prospektive klinische Studie. Unfallchirurg 104: 1134-1139

16. Philbin T, Rosenberg G, Sferra JJ (2003) Complications of missed or untreated lisfranc injuries. Foot Ankle Clin 8: 61-71

17. Pinney SJ, Sangeorzan BJ (2001) Fractures of the tarsal bones. Orthop Clin North Am 32: 21-33

18. Rammelt S, Grass R, Schikore H et al. (2002) Verletzungen des Chopart-Gelenks. Unfallchirurg 105: 371-383

19. Rammelt S, Grass R, Zwipp H (2004) Nussknackerbrüche des Kahn- und Würfelbeines. Ther Umsch 61: 451-457

20. Sanders R, Fortin P, DiPasquale A et al. (1993) Operative treatment of 120 displaced intraarticular calcaneal fractures: results using a prognostic computed tomography scan classification. Clin Orthop 290: 87-95

21. Sanders R, Swiontkowski M, Nunley J et al. (1993) The management of fractures with soft tissue disruptions. J Bone Joint Surg Am 75: 778-789

22. Thordarson DB (2000) Fractures of the midfoot and forefoot. In: Myerson MS (ed) Foot and ankle disorders, vol 2. Saunders, Philadelphia, pp 12651296

23. Zgonis T, Roukis TS, Polyzois VD (2006) Lisfranc fracture-dislocations: current treatment and new surgical approaches. Clin Podiatr Med Surg 23: 303-322

24. Zwipp H (1994) Chirurgie des Fußes. Springer, Berlin Heidelberg New York 